\title{
Notes on Stenhelmoides and description of the true male genitalia of S. strictifrons Grouvelle, 1908 (Coleoptera: Elmidae)
}

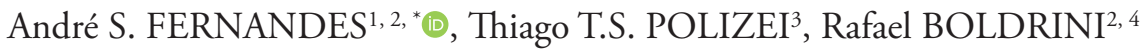 \\ ' Universidade Federal do Tocantins, Campus de Porto Nacional, Coordenação de Laboratórios de Ciências Biológicas, Laboratório de Entomologia, CP 136, \\ CEP 77500-000 Porto Nacional - TO, Brazil \\ 2 Programa de Pós-Graduação Rede de Biodiversidade e Biotecnologia da Amazônia Legal - Bionorte, Polo UFRR, CEP 69310-000 Boa Vista - RR, Brazil \\ 3 Museu de Zoologia da Universidade de São Paulo, Laboratório de Coleoptera, Av. Nazaré 481, CEP 04263-000 São Paulo - SP, Brazil \\ 4 Universidade Federal de Roraima, Campus Paricarana, Centro de Estudos da Biodiversidade, Laboratório de Entomologia, CEP 69310-000 Boa Vista - RR, Brazil \\ * Corresponding author: andresf@uft.edu.br; (D) https://orcid.org/0000-0002-5635-0291
}

\begin{abstract}
The genus Stenhelmoides comprises 15 species spread over all Central and South America and its greatest species richness is clearly associated with Amazonian regions. Based on examination of type specimens and additional material, we describe and illustrate the true male genitalia of Stenhelmoides strictifrons, which had until now been incorrectly illustrated in the literature. We also report new records of the genus Stenhelmoides from Brazil and Guyana. In addition to pictures of the species' habitus, we provide pictures of the type specimens of Stenhelmoides grandis, S. grouvellei, S. guyanensis; S. strictifrons and S. submaculus. Based on the International Code of Zoological Nomenclature, we recognize additional paralectotypes for $S$. guyanensis and for S. grouvellei.
\end{abstract}

KEYWORDS: riffle beetles, aquatic insects, morphology, Neotropics, taxonomy

\section{Notas sobre Stenhelmoides e descrição da genitália verdadeira do macho de S. strictifrons Grouvelle, 1908 (Coleoptera: Elmidae)}

\section{RESUMO}

O gênero Stenhelmoides compreende 15 espécies espalhadas por toda a América Central e do Sul e sua maior riqueza de espécies está claramente relacionada às regiôes amazônicas. Com base no estudo de espécimes-tipo e material adicional, nós descrevemos e ilustramos a genitália verdadeira do macho de Stenhelmoides strictifrons, que, até então, estava representada de maneira equivocada na literatura. Nós também reportamos novos registros de Stenhelmoides para o Brasil e Guiana. Além de imagens do habitus das espécies, fornecemos fotografias dos espécimes-tipo de Stenhelmoides grandis, S. grouvellei, S. guyanensis; S. strictifrons e S. submaculus. De acordo com as regras e recomendaçôes do Código Internacional de Nomenclatura Zoológica, reconhecemos paralectótipos adicionais das espécies $S$. guyanensis e $S$. grouvellei.

PALAVRAS-CHAVE: besouros de correnteza, insetos aquáticos, morfologia, Neotrópico, taxonomia

\section{INTRODUCTION}

Elmidae Curtis, 1830 is a cosmopolitan family of Coleoptera with around 1503 known species distributed in 151 genera (Jäch et al. 2016; Short 2018). The family has its greatest richness and abundance in the Neotropical region (Jäch and Balke 2008) and, as expected, the Amazonian elmid fauna is very diverse.

The genus Stenhelmoides Grouvelle, 1908 comprises 15 species distributed throughout the Neotropical region, from
Mexico to Paraguay (Jäch et al. 2016). Despite being more likely associated with Amazonian regions, where most of the known species can be found, the genus seems to potentially occur in many kinds of Neotropical biomes.

Grouvelle (1908) described Stenhelmoides based on two species, Stenhelmoides strictifrons and Stenhelmoides guyanensis from French Guiana (Rivière Lunier, Tumuc Humac), but without designating a type series, nor did he mention the number of specimens examined for either species. Delève, in the 1960s, examined Grouvelle's collection and designated 
types for many species, including the "holotype" for $S$. strictifrons, based on a female specimen (Spangler and Perkins 1989). Despite having labelled the specimen, Delève (1970) did not include this information in his subsequent publication about S. strictifrons. Finally, Spangler and Perkins (1989) revised the genus Stenhelmoides, and redescribed all known species, including $S$. strictifrons. They also chose a male from Grouvelle's collection, labeled similarly to the "holotype", to include in the type series as a paralectotype. They described and illustrated its genitalia.

After carefully checking specimens of Stenhelmoides strictifrons from many localities of Brazil and examining the type specimens (lectotype and paralectotype) housed in the Muséum National d'Histoire Naturelle, Paris, we concluded that the male genitalia of the species was incorrectly illustrated by Spangler and Perkins (1989). To rectify the morphological descripttion of the species and avoid potential errors by other researchers when trying to identify Stenhelmoides species, we describe and illustrate the correct male genitalia of $S$. strictifrons.

We also report new records for Stenhelmoides beebei Spangler and Perkins, 1989, Stenhelmoides stegastus Spangler and Perkins, 1989, Stenhelmoides strictifrons Grouvelle, 1908, Stenhelmoides platysternum Spangler and Perkins, 1989, and Stenhelmoides variabilis Spangler and Perkins, 1989 in Brazil, and S. stegastus and S. variabilis in Guyana. These are the first records for Stenhelmoides in the Brazilian states of Amapá, Bahia, Minas Gerais, Roraima and Tocantins. For the first time, habitus photographs of type specimens of the following species are provided: Stenhelmoides grandis Spangler and Perkins, 1989, Stenhelmoides grouvellei Pic, 1923, Stenhelmoides guyanensis Grouvelle, 1908, S. strictifrons Grouvelle, 1908, and Stenhelmoides submaculus Hinton, 1937.

Based on examination of the type series housed in the collection of the Muséum National d'Histoire Naturelle, Paris and following information provided in Spangler and Perkins (1989), Monte and Mascagni (2016), and Polizei and Casari (2019), we recognized one type specimen of $S$. guyanensis as a paralectotype and several paralectotypes of $S$. grouvellei.

\section{MATERIAL AND METHODS}

The material studied came from different Brazilian and international collections (listed below). Species level identifications were made using the taxonomic key of Spangler and Perkins (1989), original species descriptions and examination of type material. A Leica MS5 (Wetzlar, Germany) stereoscopic microscope was used for morphological studies and a Canon EOS550D (Tokyo, Japan), attached to a Leica N205A (Wetzlar, Germany) stereoscopic microscope, was used to photograph the specimens. Images were treated on Combine ZM and Adobe Photoshop.
For examination of male genitalia, the aedeagi were extracted from the specimens, cleared in heated $10 \% \mathrm{KOH}$ for five minutes (Brown 1972) and mounted on temporary slides with glycerin. Pictures were taken with the aid of a light microscope Nikon model Eclipse E200 (Tokyo, Japan), equipped with BIOPTIKA CMOS-10.0. Drawings were made on Camara Lucida and digitalized on Adobe Illustrator. Dissected genitalia were stored in microvials with glycerin attached to the same pin as the specimen.

The general morphological terminology follows Kodada $e t$ al. (2016). Specimen labels are quoted verbatim. Change of lines are indicated by "/" and different labels are separated by "//". Localities followed by “*” represent new records.

The following collections were visited and/or contain deposited specimens (curator in parentheses):

CELAMARH - Coleção Entomológica do Laboratório de Meio Ambiente e Recursos Hídricos, Universidade Federal de Goiás - Goiânia, Goiás, Brazil (Leandro G. Oliveira);

CEUFT - Coleçáo de Entomologia da Universidade Federal do Tocantins, Laboratório de Entomologia - Porto Nacional, Tocantins, Brazil (Tiago K. Krolow);

CZMT-CEMT - Setor de Entomologia da Coleção Zoológica da Universidade Federal de Mato Grosso, Departamento de Biologia e Zoologia - Cuiabá, Mato Grosso, Brazil (Fernando Vaz-de-Mello);

DZUFRJ - Coleção José Alfredo Pinheiro Dutra, Departamento de Zoologia, Instituto de Biologia, Universidade Federal do Rio de Janeiro - Rio de Janeiro, Rio de Janeiro, Brazil (Nelson Ferreira-Jr);

INPA - Coleção de Invertebrados, Instituto Nacional de Pesquisas da Amazônia - Manaus, Amazonas, Brazil (Márcio Oliveira);

MNHN - Muséum National d'Histoire Naturelle - Paris, France (Thierry Deuve);

MZSP - Museu de Zoologia da Universidade de São Paulo São Paulo, São Paulo, Brazil (Sônia A. Casari);

NHMUK - Natural History Museum - London, UK (Max Barclay).

\section{RESULTS}

Stenhelmoides Grouvelle, 1908

(Figures 1-3)

Stenhelmoides strictifrons Grouvelle 1908

(Figures 1, 3e) 
Diagnosis. (Modified from Spangler and Perkins 1989) Stenhelmoides strictifrons can be distinguished from all species of Stenhelmoides by its small size $(2.04-2.52 \mathrm{~mm})$, the dorsal plastron pattern on the pronotum, the metaventrite being slightly depressed, the elytral granules organized in rows, the absence of hair-like setae tufts on tarsi and the male genitalia with the apical surface of the parameres and median lobe densely covered with spine-like setae (Figures 1c-e).

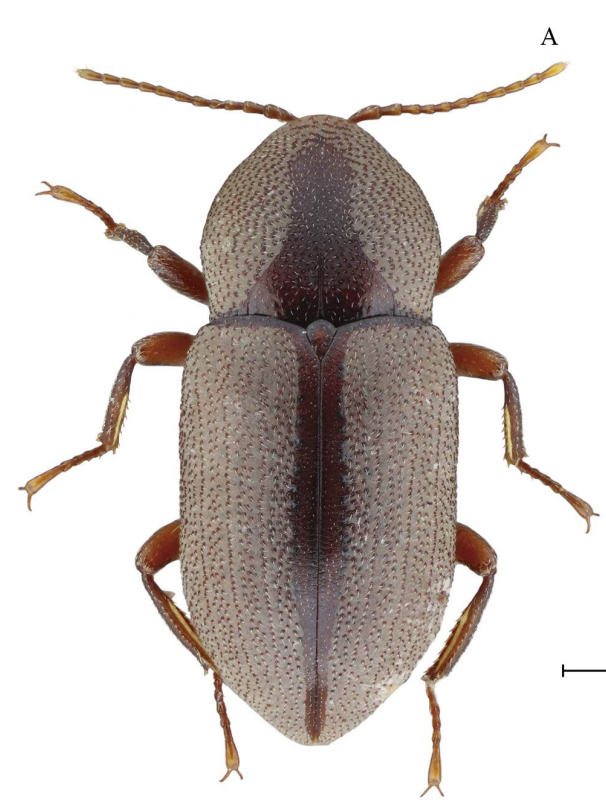

Male genitalia. Phallobase (Figures 1c, d) broad and short. In dorsal view, 1.3 times broader at base than parameres and three-fifths their length. Parameres (Figures 1c-e) as long as median lobe. In dorsal view (Figures $1 \mathrm{c}-\mathrm{d}$ ) not tapering to apex, slightly curved toward median lobe, apices feebly invaginated on internal lateral margin, rounded and covered with distinct spine-like setae over apical one-fifth. In lateral view (Figure 1e) dorsal margin nearly straight, ventral margin sinuous, apex rounded, spine-like setae concentrated on apical

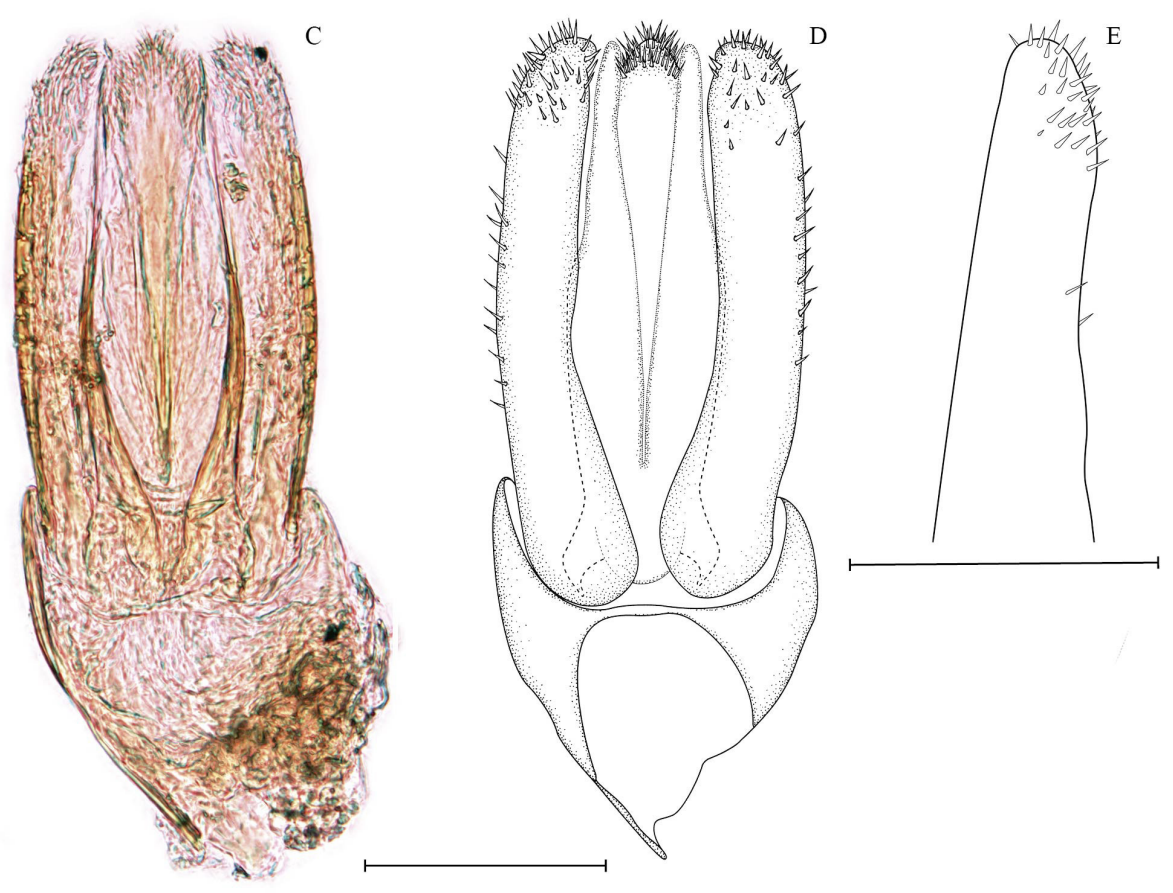

Figure 1. Stenhelmoides strictifrons Grouvelle, 1908, Male. Habitus (MZSP 31442): A - dorsal view; B - ventral view. Aedeagus: C - dorsal view; D - ventral view; E apex of paramere in lateral view. Scale bar: $(A-B)=0,5 \mathrm{~mm},(C-E)=0,1 \mathrm{~mm}$. This figure is in color in the electronic version. 
portion of ventral margin and pointed to venter. Median lobe long and richly adorned with spine-like setae. In dorsal view (Figures $1 \mathrm{c}-\mathrm{d}$ ) surrounded by dehiscent membranous structure, apex rounded and densely covered with spine-like setae thinner than those of the parameres. In lateral view completely covered by the parameres.

Type locality: French Guiana: Rivière Lunier.

Distribution: Brazil (Amapá*, Amazonas*, Bahia*, Goiás, Mato Grosso, Minas Gerais*, Pará, São Paulo, Tocantins*), French Guiana, Guyana, Peru, Venezuela.

Material examined. Lectotype (female) and paralectotype (male); $q$ // MUSEUM PARIS / GUYANE / RIVIÉRE LUNIER / TUMUC HUMAC / F.GEAY 1899 // Holotype // Muséum / Paris. (MNHN). đ // MUSEUM PARIS / GUYANE / RIVIÉRE LUNIER / F.GEAY 1899 // Type / Helmis discreta / Grouv // Paralectotype / Stenhelmoides / strictifrons / Grouvelle, 1908. (MNHN). Additional material. BRASIL. Amapá. / Tartarugalzinho. Igarapé do / Henrique. BR 156. 07.VII.2011 - / 1m. 0145'56.5”N 5052'40.5”W / A.Pes; P.Cruz \& N.Hamada leg. // Elmidae; Elminae / Stenhelmoides / T.T.S.Polizei det. 2019 // Stenhelmoides strictifrons / Grouvelle, 1908 / T.T.S.Polizei det. 2020 // Material preservado em álcool 100\% / Preservative compound alcohol 100\% (21 in INPA). (\#01) BRASIL. Amapá. Serra do / Navio. Rio Cachaço. Cachoeira / do Fernando. 02.VIII.2011 61m. / A.Pes; P.Cruz \& N.Hamada leg. / 0053'18.8”N 5201'22.9"W // Elmidae; Elminae / Stenhelmoides / T.T.S.Polizei det. 2019 // Stenhelmoides strictifrons / Grouvelle, 1908 / T.T.S.Polizei det. 2020 // Material preservado em álcool 100\% / Preservative compound alcohol 100\% (1 in INPA). BRASIL: Amazonas: Presidente / Figueiredo, Igarapé da Onça, / Sossego da Pantera, km 20, / AM-240, A. S. Fernandes / e P. V. Cruz Legs. 19/10/2008 / Armadilha Lençol Iluminado / Lâmpada Mista de Mercúrio (2 in DZUFRJ, 2 in INPA). BRASIL. Amazonas. São / Gabriel da Cachoeira. (\#04) / Paricachoeira. Igarapé Samaúma. / 1720.III.2016 Pensylvania Trap. / 00¹5’34.7”S 5947’15.3”W // Elmidae; Elminae / Stenhelmoides / T.T.S.Polizei det. 2019 // Stenhelmoides strictifrons / Grouvelle, 1908 / T.T.S.Polizei det. 2020 // Material preservado em álcool $100 \%$ / Preservative compound alcohol 100\% (1 in INPA). BRAZIL: Amazonas: Presidente / Figueiredo, Igarapé da Onça, / Sossego da Pantera, / 5951'29.7”W/202'58.3”S, / Light trap. P. V. Cruz leg. 19.x.2008 // Stenhelmoides strictifrons / Grouvelle, 1908 / André Fernandes det. (4 in INPA). BRASIL: Amazonas: Presidente / Figueiredo. Igarapé da Onça, / Sossego da Pantera, km 20 (AM- / 240). $2^{\circ} 02^{\prime}$; ; 5950'W. Luz negra / Pensilvânia trap. 4-5.VII.2000 / Ana Maria Oliveira Pes leg. // Stenhelmoides strictifrons / Grouvelle, 1908 / T.T.S.Polizei det. VI.2019 (5 in MZSP) (MZSP 42706-42710). Brasil. Bahia. Taperoá. / Opalma. Dendê. 07.I.2011 / 500m. Light trap. / Adolfo Calor et al. leg.
// Elmidae; Elminae / Stenhelmoides / T.T.S.Polizei det. 2016 // Stenhelmoides strictifrons / Grouvelle, 1908 / T.T.S.Polizei det. 2020 // Material preservado em álcool 100\% / Preservative compound alcohol 100\% // MZSP 31442 (1 in MZSP). Brasil. Bahia. Ituberá. Pratingi / 11-12.III.2011 1000m. Light trap / Adolfo Calor et al. leg. // Elmidae; Elminae / Stenhelmoides / T.T.S.Polizei det. 2016 // Stenhelmoides strictifrons / Grouvelle, 1908 / T.T.S.Polizei det. 2020 // Material preservado em álcool 100\% / Preservative compound alcohol 100\% // MZSP 31443 (1 in MZSP). BRAZIL: Goiás: Rio Verde, / 17³7'29.4”S, 51³'44.5”W, / sampling nets (2.0 mm mesh), / rocks, 7 - 11 April 2010, / A. S. Fernandes, F. F. Barbosa and L. F. R. Holanda // Stenhelmoides strictifrons / Grouvelle, 1908 (2 in CELAMARH). BRASIL. Mato Grosso. Nova / Xavantina. (\#04A) Córrego Piau. / 03.VII.2012 / 1441'00.4"S 52³6'59.4"W // Elmidae; Elminae / Stenhelmoides / T.T.S.Polizei det. 2019 // Stenhelmoides strictifrons / Grouvelle, 1908 / T.T.S.Polizei det. 2020 // Material preservado em álcool 100\% / Preservative compound alcohol 100\% (4 in INPA). BRASIL. Mato Grosso. Nova / Xavantina. Ribeirão Antártico. 05- / 06.VII.2012 (\#02) Luminosa trap / 1444'44.7”S 52²6'30.6”W // Elmidae; Elminae / Stenhelmoides / T.T.S.Polizei det. 2019 // Stenhelmoides strictifrons / Grouvelle, 1908 / T.T.S.Polizei det. 2020 // Material preservado em álcool 100\% / Preservative compound alcohol 100\% (2 in INPA). BRASIL: Mato Grosso, Cotri / guaçu. Faz. São Nicolau, / Airport, 0951'52”S, 58¹3' / 45”W, light trap, 02.x.2017, / Vaz-deMello et al. // Stenhelmoides strictifrons / Grouvelle, 1908 / T.T.S.Polizei det. 2020 (1 in CZMT-CEMT, 1 in MZSP) (CZMT-CEMT000008161) (MZSP 31439). BRASIL: Mato Grosso, / Paranaíta, 05.ix.2008 / Light Trap, N. Pinho leg. // Stenhelmoides strictifrons / Grouvelle, 1908 / T.T.S.Polizei det. 2020 (2 in CZMT-CEMT, 1 in MZSP) (CZMTCEMT000008165-000008166) (MZSP 31440). BRASIL: Mato Grosso, / Cotriguaçú, Fazenda São / Nicolau, VII.2007, Light / Trap, O. Peres Filho leg. // Stenhelmoides strictifrons / Grouvelle, 1908 / T.T.S.Polizei det. 2020 (3 in CZMT-CEMT, 1 in MZSP) (CZMT-CEMT000008162-000008164) (MZSP 31441). Sete Lagoas / MG, Brasil / III.1963, F.Werner / Martins, Silva col. // Stenhelmoides / strictifrons / Grouvelle, 1908 / T.T.S.Polizei det. 2017 // MZSP 35024 (1 male in MZSP). BRAZIL: Tocantins: Palmas: Taquaruçu / District, Fazenda Buritizal, / 48³'12.620”W 10¹7'9.548”S, / Light Trap, 27-29.iii.2018. / A. S. Fernandes leg. // Stenhelmoides strictifrons / Grouvelle, 1908 / André Fernandes det. (5 in CEUFT) (CEUFT000265-000269). GUYANA, Essequibo / Kaieteur Falls / rest house area / October 1991 // MV Light sheet / J H Martin / BMNH(E)1991-182 // Stenhelmoides / strictifrons / Grouvelle, 1908 / Thiago Polizei det. 06.2018 // MZSP 31025 (1 male in MZSP). BRASIL. Amazonas. Presidente / Figueiredo. AM 240 - Km 24. / Sítio Seu José. 20.IV.2008. / 0201'13.2”S 5949'29.1”W / Neusa Hamada 
et al. leg // Stenhelmoides strictifrons / Grouvelle, 1908 / T.T.S.Polizei det. 2020 (4 in INPA. 1 in MZSP) (MZSP 31606). BRASIL. Amazonas. Manacapuru. / AM $352-\mathrm{Km}$ 90. Sítio Sossego / do Vovô Raimundo. Igarapé / pequeno. 29.III.2008 Nessimian / et al leg. 03¹2'23"S 6040'21”W // Stenhelmoides strictifrons / Grouvelle, 1908 / T.T.S.Polizei det. 2020 ( 1 in INPA, 1 in MZSP) (MZSP 31605). BRASIL. Amazonas. Rio Preto / da Eva. AM 010 - Km 113. Ramal / Casa Branca. Igarapé da ponte. / 11.IV.2008. Pipineli et al leg. / 0247’25.2”S 59³0'10.8”W // Stenhelmoides strictifrons / Grouvelle, 1908 / T.T.S.Polizei det. 2020 (2 in INPA, 1 in MZSP) (MZSP 31607). BRASIL. Amazonas. Iranduba. I AM 352 - Km 65. Comunidade Nova / Esperança. Sítio Seu Bigode / 28.III.2008 J. Nessimian et al leg. / 0304’38.1"S 6046'11.4"W // Stenhelmoides strictifrons / Grouvelle, 1908 / T.T.S.Polizei det. 2020 (1 in INPA, 1 in MZSP) (MZSP 31604). BRASIL. Amazonas. Iranduba. / AM 352 - Km 66. Comunidade Nova / Esperança. Primeiro igarapé. / 28.III.2008 J. Nessimian et al leg. / 0305’01.4”S 60466'51.4”W // Stenhelmoides strictifrons / Grouvelle, 1908 / T.T.S.Polizei det. 2020 (1 in INPA).

\section{New records}

Stenhelmoides beebei Spangler and Perkins, 1989

(Figure 2a)

Type locality: Guyana: Mazaruni-Potaro (actually CuyuniMazaruni)

Distribution: Brazil* (Amazonas, Pará, Roraima), Guyana and Venezuela.

Material examined: BRASIL: Amazonas: Presidente / Figueiredo: Igarapé da Onça, / Sossego da Pantera, km 20 / (AM-240), 202'S, 5950'W, / Pensilvânia/Luz Negra, / A. S. Fernandes leg., 18/X/2008, // Stenhelmoides beebei / Spangler \& Perkins, 1989 / A. Fernandes det. $2009(5$ in INPA). CI. Igarapé da Ponte, / km 24 (AM - 240), 201'S, 5943'W, / Pensilvânia/Luz Negra, / A. M. O. Pes leg., 4-5/VII/2000 // Stenhelmoides beebei / Spangler \& Perkins, 1989 / A. Fernandes det. 2009 (1 in DZUFRJ). BRASIL:Para Rio / Poana a direita / Subindo 7-X-85 / Eq.Comander // Stenhelmoides beebei / Spangler \& Perkins, 1989 / T.T.S.Polizei det. 2020 // MZSP 31410 (1 in MZSP) Brasil:Roraima / Rio Uraricoera / Ilha de Maraca / 18-28-viii1987 // Stenhelmoides beebei / Spangler \& Perkins, 1989 / T.T.S.Polizei det. 2020 // MZSP 31411 (1 in MZSP).

Stenhelmoides grandis Spangler and Perkins, 1989

(Figures 2b, 3a)

Type locality: Venezuela: Amazonas
Distribution: Brazil (Amazonas, Pará*), French Guiana, Venezuela.

Material examined: BRASIL: Amazonas: Presidente / Figueiredo: Igarapé da Onça, / Sossego da Pantera, km 20 / (AM-240), 202'S, 5950'W, / Pensilvânia/Luz Negra, / A. S. Fernandes leg. 18/X/2008 // Stenhelmoides / grandis / Spangler \& Perkins, 1989 / A. Fernandes det. 2009 (7 in INPA). Pará / Cachimbo / X.955 / Pe. Perreira // Stenhelmoides / grandis / Spangler \& Perkins, 1989 / T.T.S.Polizei det. 2017 // MZSP 35023 (1 in MZSP)

Stenhelmoides platysternum Spangler and Perkins, 1989

(Figure 2c-d)

Type locality: Ecuador: Pastaza.

Distribution: Brasil* (Amazonas), Ecuador.

Material examined: BRASIL: Amazonas: Presidente / Figueiredo. Igarapé da Onça, / Sossego da Pantera, km 20 (AM- / 240). 202'S, 5950'W. Luz negra / Pensilvânia trap. 4-5.VII.2000 / Ana Maria Oliveira Pes leg. // Stenhelmoides platysternum / Spangler \& Perkins, 1989 / T.T.S.Polizei det. VI.2019 (2 in MZSP) (MZSP 42711-42712).

Stenhelmoides stegastus Spangler and Perkins, 1989

(Figure 2e-f)

Type locality: Venezuela: Bolivar

Distribution: Brazil* (Amapá, Amazonas, Mato Grosso, Pará), Guyana*, Venezuela.

Material examined: BRASIL. Amapá. / Tartarugalzinho. Igarapé do / Henrique. BR 156. 07.VII.2011 - / $1 \mathrm{~m}$. $01^{\circ} 45^{\prime} 56.5^{\prime} \mathrm{N}$ 50 52'40.5”W / A.Pes; P.Cruz \& N.Hamada leg. // Elmidae; Elminae / Stenhelmoides / T.T.S.Polizei det. 2019 // Stenhelmoides stegastus / Spangler \& Perkins, 1989 / T.T.S.Polizei det. 2020 // Material preservado em álcool $100 \%$ / Preservative compound alcohol 100\% (6 in INPA). (\#01) BRASIL. Amapá. Serra do / Navio. Rio Cachaço. Cachoeira / do Fernando. 02.VIII.2011 61m. / A.Pes; P.Cruz \& N.Hamada leg. / 0053'18.8”N 5201'22.9”W // Elmidae; Elminae / Stenhelmoides / T.T.S.Polizei det. 2019 // Stenhelmoides stegastus / Spangler \& Perkins, 1989 / T.T.S.Polizei det. 2020 // Material preservado em álcool 100\% / Preservative compound alcohol 100\% (3 in INPA). BRASIL. Amapá. (\#09) Igarapé / Tartarugalzinho. 11.VIII.2013 / 0145'55.7"S 5052'40.2"W // Elmidae; Elminae / Stenhelmoides / T.T.S.Polizei det. 2019 // Stenhelmoides stegastus / Spangler \& Perkins, 1989 / T.T.S.Polizei det. 2020 // Material preservado em álcool 100\% / Preservative compound alcohol 100\% (2 in INPA). BRASIL. Amazonas. 

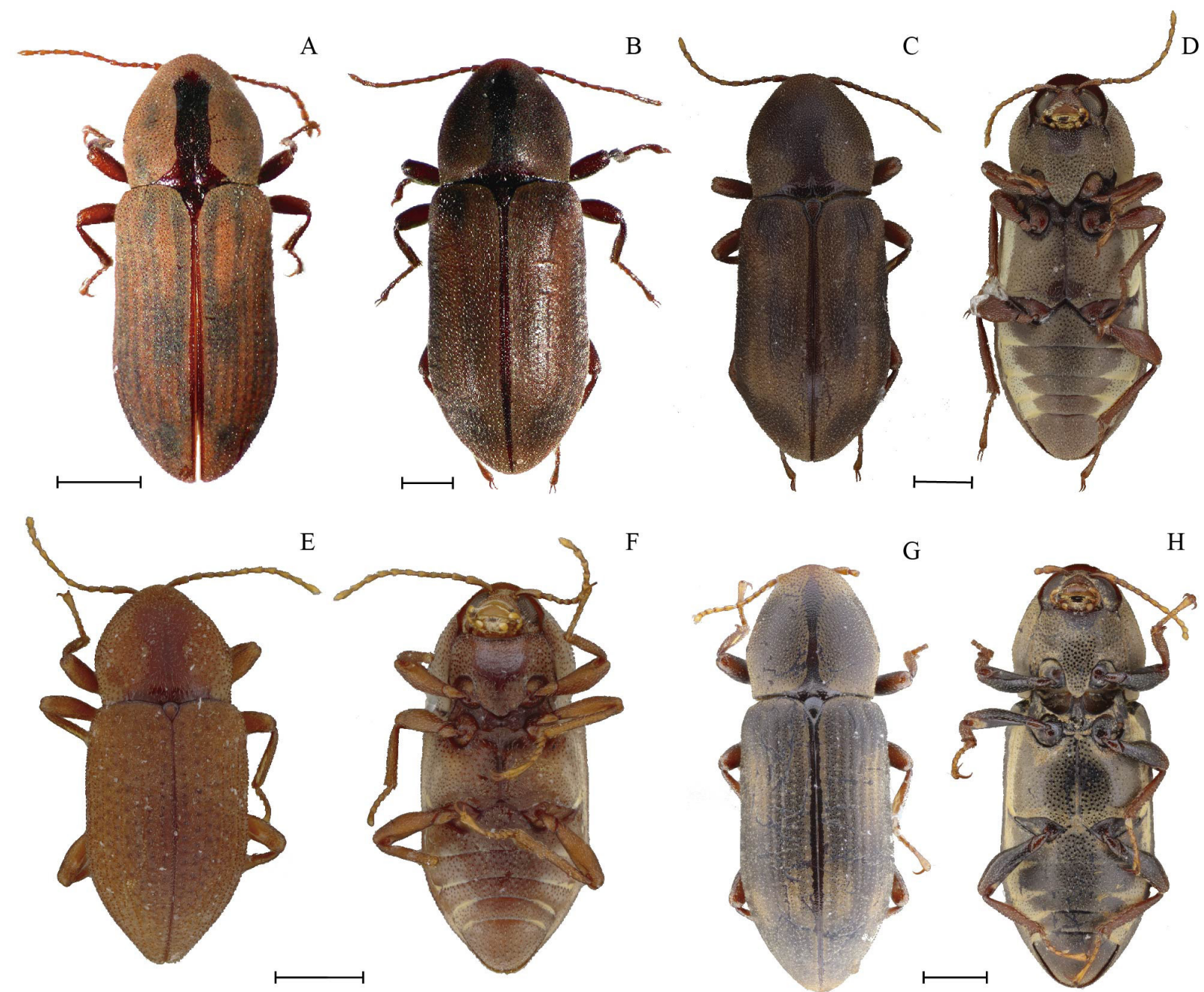

$\mathrm{F}$

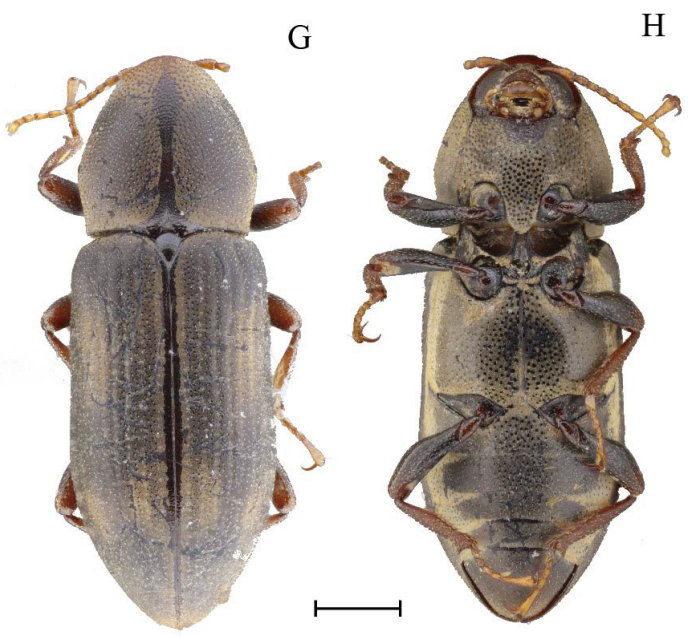

Figure 2. New photographs of Stenhelmoides Grouvelle, 1908, Habitus. A-C, E, G - dorsal view; D, F, H - ventral view. A- S. beebei Spangler \& Perkins, 1989 (INPA); B - S. grandis Spangler \& Perkins, 1989 (INPA); C-D - S. platysternum Spangler \& Perkins, 1989 (MZSP 42711); E-F - S. stegastus Spangler \& Perkins, 1989 (INPA); G-H - S. variabilis Spangler \& Perkins, 1989 (CZMT-CEMT000008175). Scale bar $=0,5 \mathrm{~mm}$. This figure is in color in the electronic version.

Apuí (\#04) / Rio Juma. Ponte BR-230 / 04.VII.2018 / 07²'43.6"S 5955'18.2"W // Elmidae; Elminae / Stenhelmoides / T.T.S.Polizei det. 2019 // Stenhelmoides stegastus / Spangler \& Perkins, 1989 / T.T.S.Polizei det. 2020 // Material preservado em álcool 100\% / Preservative compound alcohol 100\% (2 in INPA). BRASIL: Mato Grosso, / Cotriguaçú, Fazenda São / Nicolau, VII.2007, Light / Trap, O. Peres Filho leg. // Stenhelmoides stegastus / Spangler \& Perkins, 1989 / T.T.S.Polizei det. 2020 (3 in CZMT-CEMT, 3 in MZSP) (CZMT-CEMT000008167-000008169) (MZSP 31431-31433). BRASIL: Para Rio / Trombetas Cach. / Ana 12-X-85 Arm / de Luz Eq. Comander // Stenhelmoides stegastus / Spangler \& Perkins, 1989 / T.T.S.Polizei det. 2020 (10 in MZSP, 60 in INPA) (MZSP 31421-31430). BRASIL: Para Rio / Poana Acima da / Cach. Seca 9-X-85 / Eq. Comander // Stenhelmoides stegastus / Spangler \& Perkins,
1989 / T.T.S.Polizei det. 2020 (1 in MZSP, 2 in INPA) (MZSP 31412). BRASIL: Para Rio / Poana abaixo da / Cach. Seca 10X-85 / Eq. Comander // Stenhelmoides stegastus / Spangler \& Perkins, 1989 / T.T.S.Polizei det. 2020 (2 in MZSP, 2 in INPA) (MZSP 31413-31414). BRASIL: Para Rio / Poana a direita / Subindo 7-X-85 / Eq. Comander // Stenhelmoides stegastus / Spangler \& Perkins, 1989 / T.T.S.Polizei det. 2020 ( 1 in INPA) BRASIL:Para Rio / Trombetas Est. da / Fumasa Ig. da Praia / Arm de Luz 13-X-85 / Eq. Comander // Stenhelmoides stegastus / Spangler \& Perkins, 1989 / T.T.S.Polizei det. 2020 (14 in INPA, 6 in MZSP) (MZSP 31415-31420). BRASIL: Para / Rio Trombetas / Acima da Cach / do Gavião 3-X-85 / Eq. Comander // Stenhelmoides stegastus / Spangler \& Perkins, 1989 / T.T.S.Polizei det. 2020 (1 in INPA). GUYANA, Essequibo / Kaieteur Falls / rest house area / October 1991 // MV Light sheet / J H Martin / 
BMNH(E)1991-182 // Stenhelmoides / stegastus / Spangler \& Perkins, 1989 / Thiago Polizei det. 06/2018. (3 in MZSP, 2 in NHMUK) (MZSP 31028-31030).

Stenhelmoides variabilis Spangler and Perkins, 1989

(Figure 2g-h)

Type locality: Venezuela: Bolivar

Distribution: Brazil* (Mato Grosso, Roraima), Guyana*, Venezuela.

Material examined: BRASIL: Mato Grosso. / Cotriguaçú. Faz São / Nicolau. Luz.XII.2007. / O. Peres Filho // Stenhelmoides variabilis / Spangler \& Perkins, 1989 / T.T.S.Polizei det. 2020 (5 in CZMT-CEMT, 4 in MZSP) (CZMTCEMT000008170-000008174) (MZSP 31434-31437). BRASIL: Mato Grosso. / Cotriguaçú. Fazenda São / Nicolau. Luz. I.2008. / O. Peres Filho // Stenhelmoides variabilis / Spangler \& Perkins, 1989 / T.T.S.Polizei det. 2020 (1 in CZMT-CEMT, 1 in MZSP) (CZMT-CEMT000008175) (MZSP 31438). \#04A BRASIL. Mato Grosso. / Nova Xavantina. Córrego Piau. / 03.VII.2012 N.Hamada et al. leg. / 1441'00.4”S 52³6'59.4”W // Stenhelmoides variabilis / Spangler \& Perkins, 1989 / T.T.S.Polizei det. 2020 // Material preserved in $100 \%$ alcohol (1 in INPA). BRASIL, RR, Surumu / ix.1966 / M.Alvarenga \& F.M.Oliveira / col. // Stenhelmoides / variabilis / Spangler \& Perkins, 1989 / T.T.S.Polizei det. 2018. (14 in MZSP) (MZSP 30968-30982). Surumu, Roraima / IX.1966 / M.Alvarenga \& / F.M.Oliveira col. // Stenhelmoides / variabilis / Spangler \& Perkins, 1989 (7 in MZSP) (MZSP 35025-35031). GUYANA, Essequibo / Kaieter Falls / rest house area / October 1991 | MV Light sheet / J H Martin / BMNH(E)1991-182 // Stenhelmoides / variabilis / Spangler \& Perkins, 1989 / Thiago Polizei det. 06/2018. (2 in MZSP, 6 in NHMUK) (MZSP 31026-31027).

\section{Type material remarks}

Stenhelmoides grandis Spangler and Perkins, 1989

(Figure 3a)

Material examined: 1 paratype female; $\bigcirc$ // lectotype [label turned upside down to indicate its invalidity, probably done by Spangler] // MUSEUM PARIS / GUYANE / RIVIÉRE LUNIER / F.GEAY 1899 // Stenhelmoides / guyanensis / Grouv //J. Deléve det. 1967 / Stenhelmoides / guyanensis Gr. // THIS IS A NEW SPECIES, STENHELMOILDES GRANDIS / SPANGLER \& PERKINS / SEE DELEVE'S 1966 S?M. / IT BEARS A “TYPE” LABEL / \& FITS GROUVELLE'S / DESCRIPTION CORRECTLY. P.J.SPANGLER // PARATYPE / Stenhelmoides / grandis / PJ Spangler \& / PD Perkins // Muséum / Paris (MNHN).
Condition: The paratype is glued on a card and it is slightly damaged. Missing parts include the right foreleg. Ventrites IV-V and the female genitalia are glued to a card pinned below the specimen.

Comments: This exemplar was initially designated as the lectotype of $S$. guyanensis. However, in the revision of the genus, Spangler and Perkins (1989) described it as a new species $(S$. grandis) designating this specimen as one of the paratypes.

Stenhelmoides grouvellei Pic, 1923

Stenelmoides grouvellei Pic, 1923:4

(Figure 3b)

Material examined: Paralectotype (recognized here): $\delta / /$ GUYANE FRANÇSE / Nouveau Chantier / COLLECTION LE MOULT // SEPTEMBRE // MUSÉUM PARIS / Collection / Leuirole // Prepar. genit / 3766.6 // Stenhelmoides / strictifrons Grouv. / Type // J. Deléve det. 1966 / Stenelsianus / strictifrons Grouv // STENHELMOIDES / GROUVELLEI / PIC, / NOT STRICTIFRONS / AS LABELED!! / P.J.SPANGLER, 1983 // Muséum / Paris (MNHN).

Condition: The paralectotype is glued on a card and is in perfect condition. The male genitalia was extracted by Delève and mounted on a slide [Prepar. genit 3766.6].

Comments: This species was described by Pic (1923); however, he did not provide any information about the type series (syntypes), only the type locality as "Guyane Fr.”. During their revision, Spangler and Perkins (1989) found one specimen labeled as "type' (handwritten label) and three other conspecific specimens in MNHN (one without identification and two misidentified as $S$. strictifrons by Deléve in 1966). Spangler and Perkins determined all specimens as $S$. grouvellei. These three conspecific specimens were from French Guiana [very old labels]. Spangler and Perkins treated the specimen bearing the type label as the "holotype" (see Spangler and Perkins, 1989:42) designating it as the lectotype for Stenhelmoides grouvellei and the other three remaining specimens as paralectotypes (ICZN 1999, Art. 74.6). In 2017, T.T.S. Polizei found the lectotype and just one of the two paralectotypes examined by Spangler and Perkins (1989) at the MNHN. Based on the similarity of the labels and the observations of Spangler we confirmed that this specimen is one of the syntypes from Pic (1923) and is therefore a paralectotype.

Stenhelmoides submaculatus Hinton, 1937

Stenelmoides submaculatus Hinton, 1937:109

(Figure 3c) 

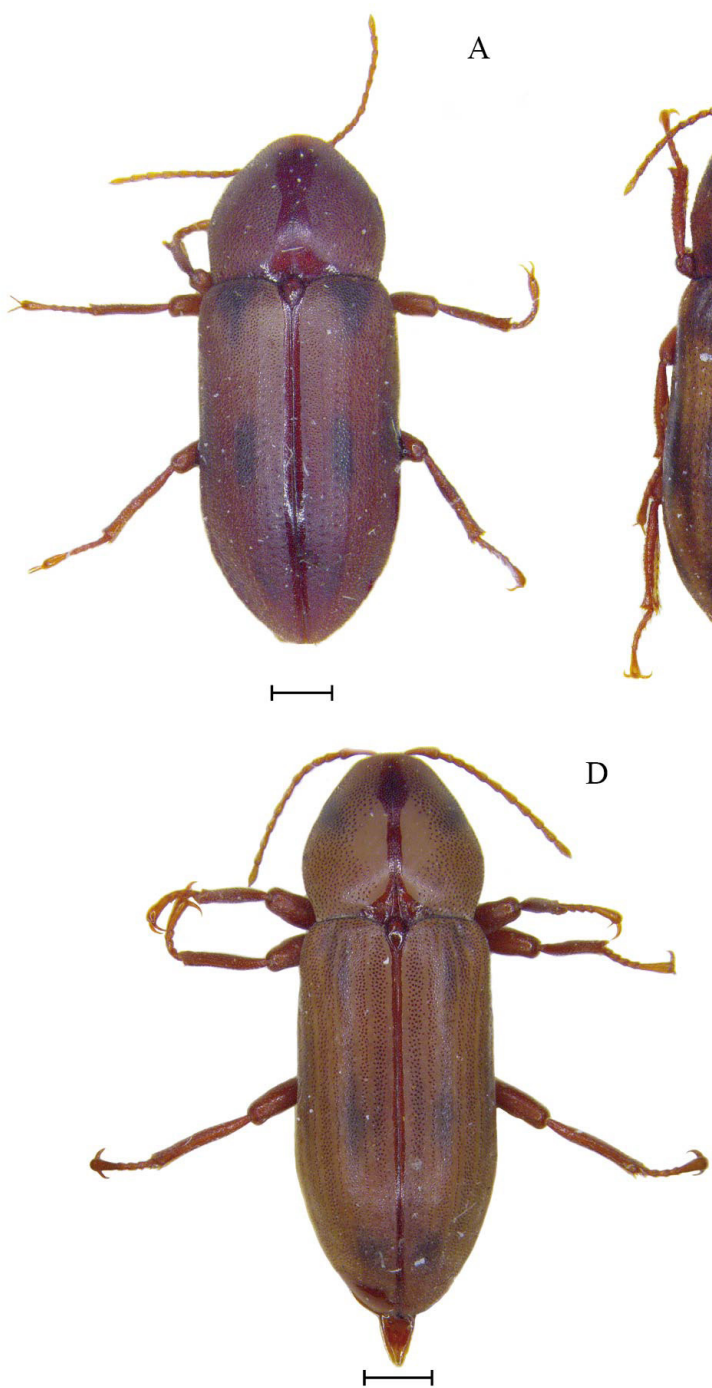

B

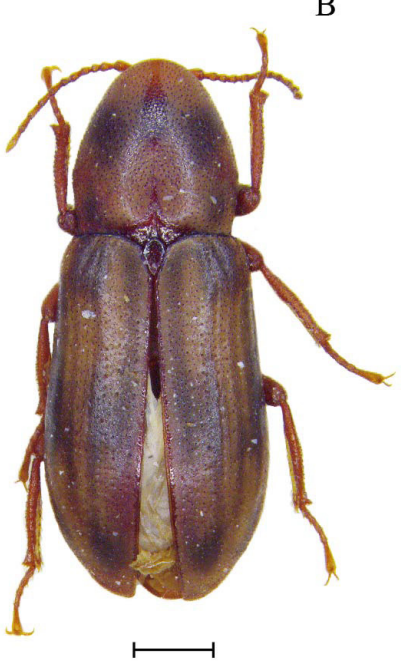

C
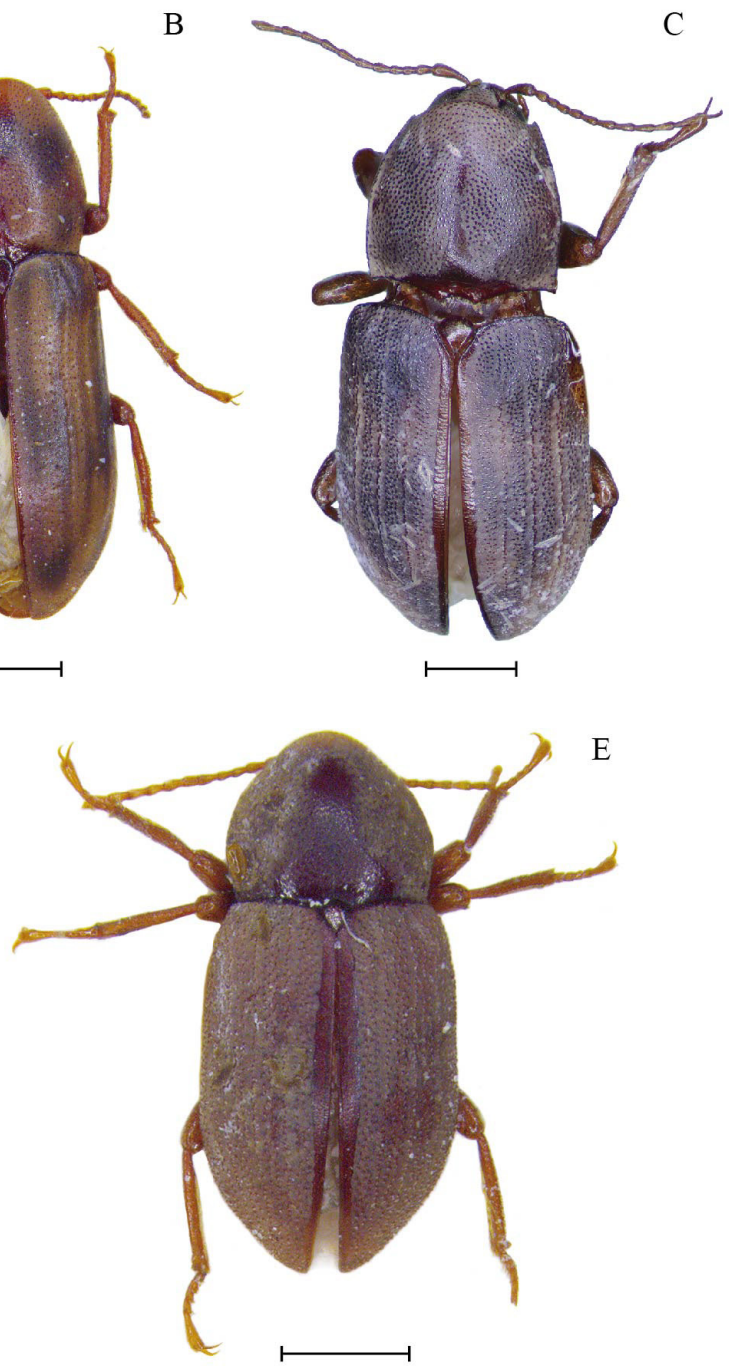

Figure 3. Types of some Stenhelmoides Grouvelle, 1908, Habitus in dorsal view. A-B, D-E - housed in MNHN; C- housed in NHMUK. A - S. grandis Spangler \& Perkins, 1989 [Paratype]; B - S. grouvellei (Pic, 1923) [Paralectotype]; C - S. submaculus Hinton, 1937 (BMNH010584113) [Paratype]; D - S. guyanensis Grouvelle, 1908 [Paralectotype]; E - S. strictifrons Grouvelle, 1908 [Lectotype]. Scale bar = 0,5 mm. This figure is in color in the electronic version.

Material examined: 1 paratype female; $q$ // Espir. Santo / x.1920-11.1921 // F.Hoffman coll. / Arp. dedic. 1921 // paratype / Stenelmoides submaculatus Hinton. // H.E.Hinton collection. / BM. 1977-566 // BMNH010584113. (NHMUK).

Condition: The paratype is glued on its right side to a triangle and it is slightly damaged. Missing parts include the two apical tarsomeres and claws on the left hind leg. Ventrites I-V and the female genitalia are glued on the same triangle as the specimen.

Stenhelmoides guyanensis Grouvelle, 1908

(Figure 3d)

Material examined: Paralectotype (recognized here): 우 // MUSEUM PARIS / GUYANE / RIVIÉRE LUNIER /
TUMUC HUMAC / F.GEAY 1899 // J. Deléve det. 1967 / Stenhelmoides / guyanensis / Grouv // Muséum / Paris (MNHN).

Condition: The paralectotype is glued on a card and it is in perfect conditions. There are no missing parts and the female genitalia are still preserved in the specimen.

Comments: In the original description of the species, Grouvelle (1908) based the species on the five specimens from Rivière Lunier et Bas-Carsevenne. However, in their revision, Spangler and Perkins (1989) studied Grouvelle's collection housed at MNHN and found just two specimens labeled as "Stenhelmoides guyanensis" by Deléve. One of these is not conspecific with the species, and Spangler and Perkins regarded it as a new species [this exemplar is one of the paratypes of $S$. grandis Spangler and Perkins, 1989, Figure 3a]. The other specimen (a female) was designated as the lectotype 
by Spangler and Perkins (1989). During a visit to the MNHN in 2017, T.T.S. Polizei found another specimen with the same data as the lectotype and also determined as "Stenhelmoides guyanensis" by Deléve in 1967 . There is no doubt that this specimen is one of the specimens that Grouvelle reported as "5 exemplars" in the original description. Besides, this species has the pronotum distinct, without granules on the disc, a condition unique among all the species of Stenhelmoides. This syntype is herewith recognized as paralectotype.

\section{Stenhelmoides strictifrons Grouvelle, 1908}

(Figure 3e)

Material examined: Lectotype; $q$ // MUSEUM PARIS / GUYANE / RIVIÉRE LUNIER / TUMUC HUMAC / F.GEAY 1899 // Holotype // Muséum / Paris. (MNHN).

Condition: The lectotype is glued on a card and it is slightly damaged. Missing parts include the apical tarsomeres and claws on the right hind leg. Ventrites I-V and the female genitalia are glued to a card pinned below the specimen.

\section{DISCUSSION}

Despite the very careful revision, for some unknown reason, Spangler and Perkins (1989) incorrectly illustrated the male genitalia of $S$. strictifrons. The error was first noticed in 2011 by A.S. Fernandes while examining the male (paralectotype) of $S$. strictifrons from Grouvelle's collection housed in the MNHN. Fernandes also discovered that Stenhelmoides specimens from different regions in Brazil he had already determined as a new Stenhelmoides species (based on the very distinct male genitalia) were actually specimens of S. strictifrons. Later, A.S. Fernandes and T.T.S. Polizei examined several specimens of $S$. strictifrons, including the lectotype (female), to confirm that the male genitalia clearly differed from those shown in Spangler and Perkins (1989).

Despite being widespread in the Neotropics and very conspicuous due to its large size, characteristic body shape and plastron pattern (Spangler and Perkins 1989; Jäch et al. 2016; González-Córdoba et al. 2020), Stenhelmoides is poorly represented in collections. Moreover, the genus is mainly known from light-trapped specimens and its larva was only discovered a few months ago (González-Córdoba et al. 2020). Most of the extant bionomic information on Stenhelmoides is provided by Spangler and Perkins (1989), who did not describe special habitat preferences or conditions associated with the specimens. Based on Spangler and Perkins (1989), Stenhelmoides specimens may be found in a variety of substrates and in different kinds of Neotropical streams. Nevertheless, during our several collections in various parts of Brazil, they were not easily found. From all the material examined in this work, only the two $S$. strictifrons specimens from Goiás state, Brazil (see Barbosa et. al. 2013) came from aquatic samples.

\section{CONCLUSIONS}

The male genitalia are one of the main morphological structures for species determination in many beetle families (Crowson 1981) and Elmidae are no different. Stenhelmoides strictifrons is the most common and widespread species of the genus in Brazil and the miss-illustrated male genitalia was a barrier to the species determination, especially for local researchers without easy access to the type specimens. By correctly illustrating the male genitalia, we enable accurate species identification for S. strictifrons. Currently, Stenhelmoides is reported from 15 countries across the Americas (Spangler and Perkins 1989; Jäch et al. 2016; González-Córdoba et al. 2020). Here we provide four new species records for the genus in Brazil, making Brazil one of the most diverse countries for the genus, alongside Venezuela, with seven known species (Jäch et al. 2016). We also increase the known distribution of Stenhelmoides in Brazil to eleven states (previously known from six), representing four of the five Brazilian geographical regions (North, Northeast, Midwest and Southeast) and three of the six Brazilian biomes (Amazon, Atlantic rainforest and Brazilian savanna) (IBGE 2019). Finally, we provided updates to the original descriptions, photographs of all Stenhelmoides species known from Brazil and valuable photographs of the type specimens for the five species to which we had access. This additional information is an important supplement to the literature, especially concerning the Brazilian Stenhelmoides fauna.

\section{ACKNOWLEDGMENTS}

We thank Dr. Antoine Mantilleri, Dr. Thierry Deuve, and Azadeh Taghavian (Muséum National d'Histoire Naturelle, France), for perfect conditions during a week stay in the Coleopteara Collection. The Coleoptera Curatorial Team of the Natural History Museum (UK) are warmly thanked for their support. We are greatful to Dr. Neusa Hamada, Dr. Jeane Cavalcante, Dr. Márcio Oliveira (Instituto Nacional de Pesquisas da Amazônia, Brazil), Dr. Leandro Oliveira (Universidade Federal de Goiás, Brazil), Dr. Fernando Vazde-Mello (Universidade Federal de Mato Grosso, Brazil), Dr. Sônia Casari (Museu de Zoologia da Universidade de São Paulo, Brazil), Dr. Adolfo Calor (Universidade Federal da Bahia, Brazil) and Dr. Tiago Kutter Krolow (Universidade Federal do Tocantins, Brazil), for providing all laboratorial conditions and assistance for collecting and/or studying the material. We thank Dr. Talita Roell (Museu de Zoologia da Universidade de São Paulo) for the support with taxonomy doubts. We also thank the reviewers and Max Barclay (NHMUK)) for their suggestions and English review. TTSP thanks FAPESP (Fundação de Amparo à Pesquisa do Estado 
de São Paulo, grants nr. 2015/17064-7 and 2016/21184-0) and CAPES (Coordenação de Aperfeiçoamento de Pessoal de Nível Superior) for scholarships.

\section{REFERENCES}

Barbosa, F.F.; Fernandes, A.S.; Oliveira, L. G. 2013. Taxonomic key for the genera of Elmidae (Coleoptera, Byrrhoidea) occurring in Goiás state, Brazil, including new records and distributional notes. Revista Brasileira de Entomologia, 57: 149-156.

Brown, H.P. 1972. Aquatic dryopoid beetles (Coleoptera) of the United States. Biota of Freshwater ecosystems identification manual, nr. 6. U.S. Environmental Protection Agency, Washington, 92p.

Crowson, R.A. 1981. The Biology of the Coleoptera. 1st ed. Academic Press, London, 814p.

Delève, J. 1970. Contribution à l'étude des Dryopoidea XX. Espèces d'Elminthidae (Coleoptera) peu ou mal connues de l'Amérique du Sud. Bulletin et Annales de la Société Royale d'Entomologie de Belgique, 106: 47-67.

González-Córdoba, M.; Martínez-Román, N.R.; Zúñiga, M.C.; Manzo, V.; Archangelsky, M. 2020. Description of the putative mature larva of the Neotropical genus Stenhelmoides Grouvelle (Coleoptera: Elmidae). Scientific Reports, 10: 1-14.

Grouvelle, A.H. 1908. Mission de M.F. Geay, dans Guyene (basin du Fleuve Carsavenne). Coléoptères: Helminthidae. Bulletin du Muséum National d'Histoire Naturelle, 14: 181-186.

Hinton, H.E. 1934. Miscellaneous Studies in the Helminae (Dryopidae, Coleoptera). Revista de Entomologia, 4: 192-201.

Hinton, H.E. 1937. Additions to the Neotropical Dryopidae (Coleoptera.). Arbeiten über morphologische und taxonomische Entomologie aus Berlin-Dahlem 4: 93-111.

IBGE. 2019. Biomas e sistema costeiro-marinho do Brasil: compatível com a escala 1:250 000. In: IBGE. (Ed.). Série Relatórios Metodológicos. v. 45. Instituto Brasileiro de Geografia e Estatística, Rio de Janeiro, 128p.

ICZN. 1999. International Code of Zoological Nomenclature. International Commission of Zoological Nomenclature, The
International Trust for Zoological Nomenclature, London, $\mathrm{xxix}+306 \mathrm{p}$.

Jäch, M.A.; Balke, M. 2008. Global diversity of water beetles (Coleoptera) in freshwater. Hydrobiologia, 595: 419-442.

Jäch, M.A.; Kodada, J.; Brojer, M.; Shepard, W.; Čiampor Jr, F. 2016. Coleoptera: Elmidae and Protelmidae. World Catalogue of Insects, v. 14, Brill, Leiden, 318p.

Kodada, J.; Jäch, M.A.; Čiampor Jr, F. 2016. 19.2. Elmidae Curtis, 1830. In: Beutel, R.G.; Leschen, R.A.B. (Ed.). Handbook of Zoology, v.IV (Part 38), Coleoptera, Beetles, v.1: Morphology and Systematics (Archostemata, Adephaga, Myxophaga, Polyphaga partim), 2nd.ed. Walter de Gruyter, Berlin, p.561-589

Monte, C.; Mascagni, A. 2016. Redescriptions and lectotype designations for two neotropical Elminae (Coleoptera: Elmidae). Zootaxa, 4175: 167-179.

Pic, M. 1923. Nouveautes diverses. Melanges Exotico-Entomologujues, 39: $1-32$.

Polizei, T.T.S.; Casari, S.A. 2019. Macrelmis pubescens (Grouvelle, 1889) -redescription of the species, designation of a lectotype and description of its larva (Coleoptera: Elmidae). Annales de la Société entomologique de France, 55: 327-338.

Spangler, P.J.; Perkins, P.D. 1989. A Revision of the Neotropical Aquatic Beetle Genus Stenhelmoides (Coleoptera: Elmidae). Smithsonian Contribution to Zoology, nr. 479, Smithsonian Institution Press, Washington, 63p.

Short, A.E.Z. 2018. Systematics of aquatic beetles (Coleoptera): current state and future directions. Systematic Entomology, 43: $1-18$.

RECEIVED: $29 / 06 / 2020$

ACCEPTED: 08/08/2020

ASSOCIATE EDITOR: Pitágoras C. Bispo 